\title{
La imagen (como) différance. Problemas y aportes
}

\author{
The image (as) différance. \\ Problems and contributions
}

\author{
JACOPO VIGNOLA \\ Universidad Alberto Hurtado (Santiago de Chile)
}

Recibido: 05/12/18 Aceptado: 07/02/19

\section{RESUMEN}

El presente trabajo se desarrolla a partir de una reconstrucción de los análisis de Roberto Rubio acerca de las dificultades en que incurre la oposición entre enfoque fenomenológico y semiótico, a la hora de aportar posibles soluciones al debate actual en filosofía de la imagen. Después de haber confrontado las perspectivas husserliana y heideggeriana con la teoría derridiana de la escritura, la referencia al arte de Duane Michals permitirá acercar el problema-imagen hacia el pensamiento de la diferencia y del acontecer diferencial, terreno icónico en donde el juego inestable e indefinido de las oposiciones produce toda posibilidad de efecto semántico.

\section{PALABRAS CLAVE:}

IMAGEN; FENOMENOLOGÍA; HERMENÉUTICA; DERRIDA; ACONTECER DIFERENCIAL

\begin{abstract}
The present article is developed from a reconstruction of Roberto Rubio's analyzes about the difficulties incurred by the opposition between phenomenological and semiotic approach, in order to providing possible solutions to the current debate on the philosophy of the image. After having confronted the Husserlian and Heideggerian perspectives with the Derridian theory of writing, the reference to the art of Duane Michals will allow us to approach the problematic
\end{abstract}

(C) Contrastes. Revista Internacional de Filosofia, vol. XXIV-N² (2019), pp. 97-113. ISSN: 1136-4076 Departamento de Filosofía, Universidad de Málaga, Facultad de Filosofía y Letras Campus de Teatinos, E-29071 Málaga (España) 
of the image towards the thought of difference and differential occurrence, iconic field where the unstable and undefined game of the oppositions produces all possibility of semantic effect.

KEYWORDS

IMAGE; PHENOMENOLOGY; HERMENEUTICS;

DERRIDA; DIFFERENTIAL OCCURENCE

\section{INTRODUCCIÓN}

EN RELACIÓN AL DEBATE ACTUAL en filosofía de la imagen, Roberto Rubio ${ }^{1}$ ha subrayado las principales debilidades que se hacen ver a la hora de encuadrar la problemática en su conjunto. Especialmente, parecería forzada y estéril la oposición entre la perspectiva fenomenológica y la semiótica, ya que, en vez de resaltar las respectivas peculiaridades, se limitaría a proponer un camino sintetizador, una especie de 'tercera vía' ausente de claridad y de una precisa diferenciación de los campos temáticos implicados. El enfoque hermenéutico, por su parte y en su declinación ontológico-heideggeriana, parece haber permitido una interpretación de la imagen en términos de experiencia no-contaminada con la esfera de lo semiótico, ni con la de la «percepción sensible». ${ }^{2}$ Bajo esta perspectiva, la apertura hacia lo ontológico haría posible profundizar más eficazmente aquellos aspectos del debate que están en juego, es decir, los conceptos de 'sentido' y de 'presencia': precisamente la relación entre éstos constituiría el mayor aporte que la perspectiva hermenéutica, frente a las dos anteriores, lograría proporcionar al debate actual. Sin embargo, Rubio nos muestra también

1 R. Rubio, «La concepción ontológica de Heidegger sobre la producción», Gregorianum, 91 (2010), pp. 343-369.

Id., «El lugar de la fenomenología en el debate de la reciente filosofía de la imagen», Veritas, 33 (2015), pp. 89-101. Id., «La reciente filosofía de la imagen. Análisis crítico del debate actual y consideración de posibles aportes», Ideas y Valores, 66.163 (2017), pp. 273-298.

2 «La reciente filosofía de la imagen. Análisis crítico del debate actual y consideración de posibles aportes», loc. cit., p. 280. No-contaminación en tanto que 'experiencia originaria', abertura a un horizonte de sentido anterior a la sensibilidad del sujeto trascendental. Se trataría de una anterioridad des-veladora de una historicidad intrínseca a la misma mostración de lo que se-da. Acontecimiento que irrumpe en el mundo - que es siempre un mundo histórico - , guardando y derramando, a la vez, una excedencia ontológica respecto, tanto a las funciones perceptivas del sujeto cognoscitivo, como a la misma temporalidad de la historia, es decir, haciendo de su presentar-se, algo fuera-del-tiempo mismo. 
una cierta dificultad, por parte del enfoque filosófico-hermenéutico, en aclarar satisfactoriamente tanto el sentido como la presencia de lo que concierne a la experiencia de la imagen; y no solamente a nivel de lo percibido/perceptible visualmente, sino también en lo que ataña al universo lingüístico. A raíz de lo anteriormente dicho, nuestro primer intento será cuestionar la legitimidad del enfoque weisingiano apoyándonos en las reflexiones desarrolladas por Rubio sobre la fenomenología de Husserl, mostrando su irreductibilidad a la perspectiva perceptualista.

A continuación, los resultados obtenidos serán confrontados con la teoría derridiana de la escritura, con el fin de averiguar si y hasta qué medida ésta pueda superar las debilidades intrínsecas tanto al enfoque semiótico como al fenomenológico. Después de haber cuestionado la teoría husserliana del conocimiento en tanto inteligibilidad intrínseca al objeto experiencial, las lentes derridianas opondrán a la teleología de la subjetividad trascendental la necesidad de ver en el objeto-imagen un movimiento constitutivamente diferencial, un inagotable diferir-de-sí-mismo, cuya captación siempre-difiriente constituye, en su indefinida posibilidad de repetir-se, la condición de toda vivencia posible.

En tercer lugar, la reconstrucción temática del enfoque hermenéutico especialmente el desarrollado por Heidegger - será confrontada con el pensamiento deconstructivo del filosofo francés, para así abrirnos paso hacia una estética de la visión capaz de superar la dicotomía entre auténtico e inauténtico, entre originario y derivado. Finalmente, la referencia al arte de Duane Michals nos permitirá trasladar la problemática en su conjunto, desde la comprensibilidad y el aparecer con sentido hacia el pensamiento de la diferencia y del acontecer diferencial, es decir - como tematizaremos - hacia aquel terreno icónico en donde el juego inestable e indefinido de las oposiciones produce toda posibilidad de efecto semántico.

\section{LA FILOSOFÍA DE LA IMAGEN ENTRE FENOMENOLOGÍA Y DIFFÉRANCE}

En la medida en que nos proponemos trasladar el núcleo teórico del debate hacia el terreno fenomenológico, la pregunta trascendental concerniente a la filosofía de la imagen, resulta ser: «¿cómo es que una superficie visible puede remitir hacia algo que no está allí en persona y hacerlo presente?». ${ }^{3}$ Respecto a las dos posibles respuestas, es decir, referencia «hacia algo» en calidad de «signo», por un lado, $\mathrm{y}$ «experiencia derivada de la percepción sensible», ${ }^{4}$ por otro, se suele atribuir este segundo enfoque a la fenomenología. Criticando tal atribución, Rubio nos muestra que la recepción del debate por parte de Lambert

3 «El lugar de la fenomenología en el debate de la reciente filosofía de la imagen», loc. cit., p. 90.

4 Ibid.

Contrastes vol. XXIV-N² (2019) 
Wiesing ${ }^{5}$ trata de dar cuenta del supuesto antagonismo de estas perspectivas entendiéndolas, a nivel de exhibición en imagen, por un lado, como orientación hacia $-\mathrm{y}$ mediante - el sentido y, por otro, como presentación sensible. $\mathrm{Si}$, en el primer caso, tendríamos un enfoque atento a la función significante y referencial de la imagen, en el segundo encontraríamos la dimensión de la presencia en tanto que dar-se inmediato del objeto.

El problema de esta segunda perspectiva interpretada por Wiesing radicaría en que, limitándose el carácter exhibitorio de la experiencia icónica únicamente al momento del objeto-imagen, pasaría por alto la relación entre éste y el tema de la imagen - su sujet - y, al mismo tiempo, ignoraría lo que posibilita a la misma conciencia de la imagen en la percepción.

En el planteamiento wiesingiano, el objeto-imagen gozaría de una independencia onto-fenomenológica, mientras que, según Rubio, no se trataría más que de una modalidad, por parte del tema-imagen, de hacerse 'accesible' a la experiencia. A este nivel del discurso, se hace necesario remitirnos a la fenomenología husserliana, sea porque es Husserl el pensador al cual se refiere Rubio en sus argumentaciones para poner en tela de juicio la equiparación wiesingiana entre enfoque perceptualista y perspectiva fenomenológica, sea - y precisamente - porque tal referencia nos ofrecerá el enlace para reflexionar acerca de la teoría derridiana de la escritura en aquellos aspectos que, de manera más significativa, puedan confrontarse con los nudos problemáticos de la filosofía de la imagen, en relación a las líneas teóricas del así llamado 'giro icónico'. ${ }^{6}$

Según Rubio, en la teoría husserliana del conocimiento no hay objeto de experiencia que prescinda de una dimensión significativa: todo objeto experiencial se nos da con sentido, sea o no sea el objeto mismo existente. Paralelamente, el aparecer del objeto sigue manteniendo una cierta inteligibilidad independientemente de su grado de presencia, es decir, sea el objeto presente o no-presente a la intuición correspondiente. En el primer caso, hablaríamos de actos «presentativos», es decir, «actos en los cuales el objeto intencional aparece con algún grado de presencia»; en el segundo caso, se trataría de actos meramente «signitivos», actos que «hacen accesible a su correspondiente objeto», «sin tenerlo presente en grado alguno»». ${ }^{7}$

Ahora bien, la síntesis entre estos dos actos es precisamente lo que, en Husserl, posibilita al conocimiento mismo y, a la vez, permite asegurar

5 L. Wiesing, Phänomene im Bild. München: Fink, 2000. Id., Artifizielle Präsenz. Studien zur Philosophie des Bildes. Frankfurt a. M.: Suhrkamp, 2005.

6 Sobre el concepto y la problemática del 'giro icónico', véase, entre otros, A. García Varas, «Imágenes, cuerpos y tecnología: dos versiones del giro icónico», Thémata. Revista de Filosofia, 46 (2012), pp. 717-730.

7 «El lugar de la fenomenología en el debate de la reciente filosofía de la imagen», loc. cit., p. 94. 
«el carácter no arbitrario del conocer». En la medida en que el concepto de intencionalidad logra sobrepasar los límites del psicologismo, hace de este sobrepaso la condición de posibilidad misma de la experiencia, permitiendo que «el objeto de conocimiento» constituya «el correlato directo de vivencias que estructuralmente están dirigidas a objetos» ${ }^{8}{ }^{8}$ Podemos, pues, distinguir entre conocimiento-en-potencia y conocimiento-en-acto: ${ }^{9}$ si el primero se refiere semánticamente al nivel de lenguaje, el segundo concierne a la unión sinérgica entre sentido del objeto y su presencia, es decir, la síntesis entre la mera inteligibilidad del objeto y su efectivo dar-se a la intuición. La experiencia icónica del sujeto cognoscitivo será entonces aquella dimensión en la que siempre habrá presencia de imagen, aunque, en su intensidad más baja, se limitará a la modalidad de la conciencia, mientras, en su presencia plena, constituirá una percepción sensible en cuanto tal. Hay, por lo tanto, una heterogeneidad intensiva del hacerse-presente-de-la-imagen, ya que, a nivel de «conciencia de imagen, el objeto intencional aparece con sus elementos sensibles propios, pero no de manera íntegra». ${ }^{10}$

La complejidad experiencial en la que actúa la conciencia de imagen husserliana viene subrayada por Rubio a partir de dos razones principales: la primera se halla en que nos enfrentamos a «tres orientaciones intencionales» distintas, pero, a la vez, íntimamente entrelazadas, es decir, «hacia la cosaimagen, el objeto-imagen» y el tema de la imagen representado en ella, «el sujet-imagen»». ${ }^{11}$

El segundo factor de complejidad reside en la dimensión doblemente conflictual de estas orientaciones, ya que, por un lado hay conflicto entre la dirección hacia la cosa-imagen y el objeto-imagen. Por otro lado, la relación dialéctica concierne también al objeto-imagen frente al tema de la imagen misma.

Al mostrar la irreductibilidad del enfoque fenomenológico a la perspectiva perceptualista, los trabajos de Rubio nos permiten también vislumbrar la

\section{Ibíd.}

9 Me refiero aquí al movimiento mismo de la plenificación en sus crecientes gradaciones de presencia a la intuición. La distinción entre 'en-potencia' y 'en-acto' es entendida, pues, en términos estrictamente dinámicos, es decir, ni dicotómicos, ni sustanciales (no nos referimos a dos realidades distintas). Más que de dos tipos de conocimiento, querría hablar de una misma actividad conciencial, intencionalmente dirigida a la significación del objeto empírico. Este constitutivo - e imprescindible para Husserl, en mi opinión - 'dirigirse-hacia' de la intencionalidad hace que el conjunto de las gradaciones significativas se ordene progresivamente, de manera que la sucesión de las anteriores preparen la de las posteriores, haciendo de éstas el cumplimiento de aquéllas.

10 Ibid., p. 95.

11 Ibid., p. 96. 
necesidad de desarrollar otras argumentaciones, de abrir distintos caminos de investigación en torno al pensamiento de la experiencia icónica. A raíz de ello, nos preguntamos: ¿puede la teoría derridiana de la escritura aportar algo nuevo a los planteamientos aquí descritos? Mi opinión que sí, que puede hacerlo, aunque no para resolver dudas, sino más bien añadiendo articulaciones problemáticas. Éstas pueden vislumbrarse ya a partir de una confrontación con la fenomenología de Husserl: la importancia otorgada por Derrida a la escritura, a su inagotable legibilidad, la cual asegura que un texto pueda leerse indefinidas veces, independientemente de los distintos contextos, refleja lo que, a nivel fenomenológico, Husserl concibe y defiende en su teoría del conocimiento como inteligibilidad intrínseca del objeto experiencial, en la medida en que, nos recuerda Rubio, «todo objeto aparece en la experiencia de manera significativa, es decir, con sentido». ${ }^{12}$

Se trata de una inteligibilidad que Husserl extrae de la dimensión estructuralmente intencional de la actividad cognoscitiva y que le lleva a concebir - subraya Rubio - «a la intencionalidad como la estructura básica de la experiencia con valor epistémico, en la medida en que el objeto de conocimiento es el correlato directo de vivencias que estructuralmente están dirigidas a objeto».. ${ }^{13}$ Pues bien, precisamente esta actitud fenomenológica se ve cuestionada por Derrida en tanto que expresión de un cierto «platonismo» que, a nivel semántico, hace depender el significado de lo que se enuncia y/o se da a la intuición, exclusivamente en el acto intencional, de manera tal que su contenido resulte un ideal en sí mismo a partir de la intención subyacente al acto significante.

La crítica a la intencionalidad es coherente con la puesta en tela de juicio de la relación husserliana entre «verdad»y «evidencia», relación que es imprescindible para la intuición de esencias capaces de mostrarse autónomamente, es decir, en calidad de fenómenos no-materiales que gocen de existencias independientes frente a toda clase de materialidad. Husserl nos hablaría de un objeto - a nivel de fenomenología de la imagen - y de una verdad - a nivel de teoría del conocimiento - que se auto-dan, que se nos ofrecen por-sí-solas pasando por la dimensión intencional de nuestros actos intuitivos, en la medida en que habría una relación directa entre el acto portador de la intención semántica y el acto que realiza a ésta, de la misma manera en que la representación de un objeto se relaciona con la actualidad de su percepción. Pues bien, Derrida vislumbra, en este proceder, el alma platónica del discurso fenomenológico, es decir, una práctica filosófica proyectada hacia una metafísica de la presencia y de la identidad del significado consigo mismo, a través de un intuicionismo fenomenológico que, si por un lado, hace que el conocimiento esté, de acuerdo a

12 Ibid., p. 94.

13 Ibid.

Contrastes vol. XXIV-N² (2019) 
Rubio, «orientado teleológicamente hacia el ideal de la máxima plenificación», ${ }^{14}$ por otro, acaba encerrándose en una subjetividad trascendental que no consigue ver, en lo que no-está-todavía-presente, el efecto espacio-temporizador de lo que está presente-en-su-ausencia, de lo que, aplazando su aparición, fundamenta la misma presentación de lo que se-da. El acto intuitivo del objeto-imagen es, para Derrida, intrínsecamente diferencial, nunca refleja un objeto idéntico a sí mismo, ya que éste está enredado en el juego de las protenciones y retenciones husserlianas, las cuales nos obligan a reconocer el movimiento del «diferir» en tanto que «temporización» y «espaciamiento», es decir, el «hacerse tiempo del espacio» y el «hacerse espacio del tiempo». ${ }^{15}$

Al objeto idéntico a sí mismo, cuya identidad viene otorgada por la plenitud de la actividad intuitiva, Derrida opone la no-catalogable pluralidad semántica de la différance:

La diferencia es lo que hace que el movimiento de la significación no sea posible más que si cada elemento llamado «presente», que aparece en la escena de la presencia, se relaciona con otra cosa, guardando en sí la marca del elemento pasado y dejándose ya hundir por la marca de su relación con el elemento futuro, no relacionándose la marca menos con lo que se llama el futuro que con lo que se llama el pasado, y constituyendo lo que se llama el presente por esta misma relación con lo que no es él: no es absolutamente, es decir, ni siquiera un pasado o un futuro como presentes modificados. ${ }^{16}$

Este movimiento espacio-temporizador de la différance encuentra sus puntos de reparo ya en La voz y el fenómeno, al cuestionar la conciencia interna del tiempo analizada por Husserl, y su consiguiente pasar por alto la ineludible diferencia temporal en la que queda implicada toda vivencia y que, en su alteridad, hace a ésta posible:

Desde el momento en que se admite esta continuidad del ahora y del no ahora, de la percepción y de la no percepción en la zona de originariedad común a la impresión originaria y a la retención, se acoge lo otro en la identidad consigo del Augenblick: la no-presencia y la inevidencia en el parpadeo del instante. Hay una duración del parpadeo; y ella cierra el ojo. Esta alteridad es incluso la condición de la presencia, de la presentación y, en consecuencia, de la Vorstellung en general, antes de todas las disociaciones que podrían producirse. ${ }^{17}$

14 Ibid., p. 97.

15 J. Derrida [1972a], Márgenes de la filosofía, tr. Carmen Gonzáles Marín. Madrid: Cátedra, 2017, p. 43.

16 Ibid., p. 48.

17 Id. [1967a], La voz y el fenómeno. Introducción al problema del signo en la fenomenología de Husserl, tr. Patricio Peñalver. Valencia: Pre-Textos, 1985, p. 119. 
Bajo esta perspectiva, para que la vivencia husserliana pueda darse en la intuición y re-presentarse en la reflexión, necesita a una originaria «no-identidad consigo» misma, la cual no puede pensarse más que a partir de la différance en tanto movimiento mismo del diferir, es decir, movimiento en calidad de pliegue anterior a toda presencia y que, en su infinita posibilidad de repetir-se, constituye la misma condición de toda vivencia posible:

Sin esta no-identidad consigo de la presencia llamada originaria, ¿cómo explicar que la posibilidad de la reflexión y de la re-presentación pertenezca a la esencia de toda vivencia? [...] Husserl lo subraya sin cesar, para la reflexión, sobre todo en Ideas I, y para la re-presentación ya en Lecciones. En todas estas direcciones, la presencia del presente es pensada a partir del pliegue del retorno, del movimiento de la repetición, y no a la inversa. Que este pliegue sea irreductible en la presencia o en la presencia a sí, que esta huella o esta diferancia sea siempre más vieja que la presencia, y le procure su apertura, ¿no prohíbe todo eso hablar de una simple identidad consigo mismo «im selben Augenblick»? ${ }^{18}$

En tanto movimiento espacio-temporizador de la temporalidad presente, la diferancia es, a la vez, archi-escritura - escritura originaria e independiente respecto al sujeto que escribe - y lo que, en la imagen, constituye su acontecer diferencial, ya que, así como su captación es siempre diferente, ella misma produce diferencia(s).

A nivel fenomenológico, la experiencia de imagen se revela muy compleja, especialmente, en la versión husserliana analizada por Rubio, donde por «conciencia de imagen» tenemos que entender «una experiencia compleja de intencionalidades en conflicto», por un lado, «entre la intencionalidad dirigida hacia la cosa-imagen y la intencionalidad dirigida al objeto-imagen»y, por otro, «entre la intencionalidad del objeto-imagen y la intencionalidad dirigida al sujet-imagen». ${ }^{19}$

En este sentido, el enfoque fenomenológico-husserliano no se agota en las fronteras teoréticas de la perspectiva perceptualista, aunque ambas puedan considerarse pertenecientes a una raíz común que remonta al imprescindible momento del objeto-imagen. Éste, sin embargo - y en línea con Rubio - no tiene una autonomía onto-fenomenológica tal como para poder prescindir de su conexión con el «sujet-imagen», ya que constituye, como habíamos adelantado arriba, una modalidad de su aparición y accesibilidad - aspecto que quedaría ignorado por Wiesing.

18 Ibid., p. 122.

19 «El lugar de la fenomenología en el debate de la reciente filosofía de la imagen», loc. cit., p. 96. 
Con todo, hemos visto que las reflexiones derridianas al respecto, si por un lado tienen en cuenta la complejidad del enfoque husserliano, por otro, no pueden sino desarrollarse a partir de una deconstrucción de la intencionalidad, tanto a nivel de objeto experiencial como a nivel de actividad significadora llevada a cabo por una subjetividad trascendental teleológicamente orientada hacia una plenitud intuitiva siempre idéntica a sí misma. La experiencia icónica implica un intrínseco movimiento espacio-temporizador que se refleja en la posibilidad, por parte de la imagen misma, de mostrarse siempre diferante, es decir, nunca idéntica a sí misma y siempre capaz de producir diferencia(s).

Bajo este punto de vista, el enfoque semiótico presentado por Wiesing 20 no parece tener menores dificultades a la hora de confrontarse con la deconstrucción derridiana de la pareja significante/significado. Ésta, para el filosofo francés, no sería más que el testimonio de un logocentrismo que ha acompañado a la historia del pensamiento occidental hasta el mismo Heidegger y que implica una «relación de traducción o de significación natural» entre «el ser y el alma», así como, «entre el alma y el logos» supondría «una relación de simbolización convencional». ${ }^{21} \mathrm{La}$ distinción entre significante y significado es íntimamente metafísica, ya que refleja - y no puede prescindir de - la «diferencia entre lo sensible y lo aquí inteligible».

Toda referencia a un significado remitiría «a un logos absoluto al cual está inmediatamente unido» y que, en la «teología medieval» constituía «una subjetividad creadora infinita» orientada «hacia el lado del verbo y de la cara de Dios». ${ }^{22}$ De todas formas, nos recuerda Derrida, el hecho de que la «época del signo» sea «esencialmente teológica» no hace que la noción misma de «signo» - y la consiguiente diferencia entre significado y significante - deje de ser necesaria en determinados contextos. Es la idea de un signo independiente de la escritura, la idea de un «significado trascendental» lo que resulta rechazado por el filosofo francés. La escritura es anterior a todo signo, aunque haya sido siempre subordinada a la dimensión fonética del logos, a una racionalidad preconstituida unida inmediatamente a la «voz de la conciencia»:

El concepto de signo (significante/significado) contiene en sí mismo la necesidad de dar preeminencia a la sustancia fónica y de erigir la lingüística como "modelo" de la semiología. La phonè es la sustancia significante que se da en la conciencia

20 «La reciente filosofía de la imagen. Análisis crítico del debate actual y consideración de posibles aportes», loc. cit., pp. 273 ss.

21 J. Derrida [1967b], De la gramatología, tr. Oscar del Barco y Conrado Ceretti. Colombia: Siglo XXI, 1986, p. 17.

22 Ibid., p. 20. 
más íntimamente ligada al pensamiento del concepto significado. Desde este punto de vista, la voz es la conciencia misma. ${ }^{23}$

Derrida ha producido «un nuevo concepto de escritura», en virtud del cual no puede haber algún elemento fónico o gráfico que no remita inmediatamente a otro, en un juego de cadenas diferenciales en continuo devenir, y cuyos componentes se escapan de la oposición presencia/ausencia. El elemento de dicha escritura es el «grama como différance», es decir, «el juego sistemático de las diferencias, de las huellas de diferencias, del espaciamiento mediante el cual los elementos se relacionan unos con otros». Es precisamente este «devenirespacio» de los elementos fónicos y gráficos lo que permite que haya escritura y que se realice «cualquiera correspondencia entre el habla y la escritura, cualquier tránsito de una a otra». ${ }^{24}$

Bajo esta perspectiva, el juego de las diferencias es anterior a cualquier elemento lingüístico - sea hablado o escrito - así como a las parejas sujeto/ objeto, activo/pasivo, ideal/material, ya que la relación misma entre estos términos no es más que «un efecto de différance, un efecto inscrito en un sistema de différance». ${ }^{25}$ Consiguientemente, la misma oposición entre el significante y el significado, en la medida en que pretenda revindicar un sentido anterior a la différance, se vuelve contradictoria.

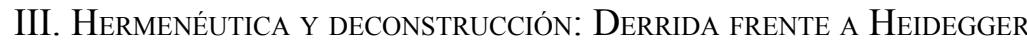

Algunos motivos teóricos sugestivos para otros posibles aportes al presente debate han de encontrarse también en el enfoque hermenéutico, el cual, desarrollándose a partir de la fenomenología, se orienta «hacia el aparecer en la experiencia» y deriva de ello que «ser es aparecer con sentido», es decir, aparecer envuelto y garantizado por una intrínseca «dimensión de comprensibilidad». ${ }^{26}$

Ahora bien, tal aparecer con sentido constituye un proceso interpretativo estructurado por múltiples factores - el objeto de lo que aparece, el contexto de la interpretación, los sujetos que interpretan - que interactúan entre sí, haciendo de la imagen un tipo peculiar de experiencia, o mejor dicho, de acontecimiento, en virtud del cual - nos recuerda Rubio - lo importante «no son los detalles empíricos», «sino más bien las características estructurales del proceso de génesis de sentido que tienen lugar en la experiencia icónica». ${ }^{27} \mathrm{El}$ enfoque

23 Id. [1972b], Posiciones. Entrevista con Jacques Derrida, tr. y prólogo Manuel Arranz. Valencia: pre-Textos, 2014, p. 41.

24 Ibid., pp. 47-48.

25 Ibid., p. 50.

26 «La reciente filosofía de la imagen. Análisis crítico del debate actual y consideración de posibles aportes», loc. cit., p. 283.

27 Ibid., p. 284. 
hermenéutico - especialmente el desarrollado por Heidegger - aborda el problema-imagen confrontándose críticamente con aquella tradición ontológica centrada en el dualismo entre sensible e inteligible, material e ideal, empírico y a priori, y que está animada por tres hitos principales.

Relativamente al primero, no habría aparición sensible sino en tanto que «la imagen de un ítem ideal o de una Idea». El segundo remonta a Kant y asigna a la imaginación la doble actividad de «hacer presente sensiblemente algo que no está» y actuar como puente entre lo empírico y lo a priori. El tercer hito se basa en la capacidad, para la expresión artística, de presentar a lo sensible sin que «haya separación entre la aparición» en cuanto tal y «lo presentado en ella». ${ }^{28}$

Rechazando el primer planteo, Heidegger parece acercarse a la concepción kantiana según la cual exhibir significaría hacer sensible, poner-en-vista, hacer que la imagen resulte sensiblemente accesible. Bajo esta perspectiva, el filosofo alemán estaría pensando en una imaginación capaz de ofrecer una exhibición ontológicamente auto-suficiente, independiente de las contingencias sensibles y, a la vez, en calidad de acontecimiento extra-ordinario que irrumpe en ellas, confiriendo sentido a la materialidad de lo que está puesto-en-obra. El discurso heideggeriano no puede sino orientarse hacia el arte, entendiendo a éste, esencialmente, como producción de lo que aparece, como salida a la luz de lo que antes estaba oculto. ${ }^{29}$ Es interesante notar que este aproximarse a la luz - de acuerdo a Rubio - encuentre siempre una fuerza opositora, una resistencia, podríamos casi decir 'una fuerza igual y contraria' que empuja para que lo que se está des-ocultando permanezca en la dimensión de lo oculto. Precisamente en este pólemos, en este conflicto heraclíteo se hallaría la esencia del arte en tanto acontecimiento a-lethéico. Mediante este planteamiento, el enfoque ontológico-hermenéutico logra que los conceptos de «presencia» y «sentido» adquieran una fisionomía nueva, ya que sus dinámicas generadoras no conciernen a propiedades físico-materiales, ni a las posibles modalidades de empleo y funcionalidad del objeto-imagen. Vislumbramos aquí, más bien, «el surgimiento, siempre incipiente, de sentido y presencia sensible que irrumpe contra el estilo de la experiencia habitual, y también contra el orden de la experiencia cognoscitiva». ${ }^{30}$ Concordamos, pues, con Rubio en reconocer la irreductibilidad del enfoque hermenéutico a una especie de 'tercera vía' o de camino intermedio entre semióticos y perceptualistas.

28 Ibid., p. 285.

29 Cf. M. Heidegger [1977], El origen de la obra de arte. Caminos de bosque, tr. Helena Cortés y Artuto Leyte. Madrid: Alianza Editorial, 2012.

Véase también R. Rubio, «La concepción ontológica de Heidegger sobre la producción», loc. cit.

30 «La reciente filosofía de la imagen. Análisis crítico del debate actual y consideración de posibles aportes», loc. cit., p. 292. 
La peculiaridad de la perspectiva heideggeriana puede aportar nuevas sugerencias al problema en torno a la experiencia de imagen, implicando una profundización ulterior de los conceptos de «sentido» $\mathrm{y}$ «presencia» $\mathrm{y}$, a la vez, des-velando un campo energético de fuerzas-en-conflicto, en virtud del cual lo icónico se nos revela como experiencia del acontecimiento, irrupción de lo extra-ordinario en lo habitual y corriente.

Ahora se trata de individualizar aquellos nudos temáticos donde la concepción derridiana de la escritura pueda confrontarse de la manera más significativa y fecunda con el enfoque hermenéutico aquí teorizado. Tomando apoyo en Derrida, intentaremos llevar a cabo un cuestionamiento de los conceptos de 'presencia', 'sentido', 'exhibición originaria', utilizados por Heidegger, para luego ver cómo estas mismas expresiones pueden abordarse desde una perspectiva radicalmente distinta a la desarrollada por la historia de la metafísica, hasta el mismo Heidegger incluso. En su propósito de crítica radical de la «ontología tradicional», el filosofo alemán no ha podido evitar relacionarse problemáticamente con el concepto de «tiempo», ya que el existente es, en términos ónticos, el ente presente y, a nivel ontológico, el ser en tanto que presencia. Consiguientemente, la determinación óntica del ser concierne a una modalidad temporal determinada que es la del «presente»y, a nivel histórico-filosófico, según Derrida, el ser y el tiempo no han podido pensarse más que «a partir del presente, en la forma del presente», es decir, más que recurriendo al concepto de "presencia», ${ }^{31}$ al cual tampoco Heidegger habría podido renunciar. En la perspectiva de Sein und Zeit, ${ }^{32}$ el hacerse-presente de la imagen, en tanto que des-ocultamiento de lo oculto, hace que la presencia del presente esté, paradójicamente, fuera-del-tiempo: la verdad de lo que se hace presente es, en este sentido, eterna, no está sujeta a la temporalidad, aunque acontezca en un cierto ahora fenomenológico.

Pareciera que la eternidad de la presencia, también en Heidegger, tendría una causa teleológica, en la medida en que el hacerse-presente de la presencia es el momento de la presencia-en-acto. Ahora bien, en tanto que realización de lo que pod(r)ía-ser, en su efectivo ser-presente, la presencia-en-acto constituye, a la vez, el comienzo y el fin del acontecimiento, es decir, su principio - origen temporal - y su causa final, y el desvelamiento de esta verdad sería precisamente lo que nos hace experimentar la imagen-puesta-en-obra. Según el filosofo francés, la presencia, en tanto que «sentido» o «esencia de lo que es», adquiere, de esta manera, un carácter divino:

31 Márgenes de la filosofía, op. cit., p. 65.

32 M. Heidegger [1979], Ser y Tiempo, tr. Jorge Eduardo Rivera. Madrid: Trotta, 2009. 
El Primer Motor, como «acto puro» (energeia è kath' auten) es presencia pura. En tanto que tal, anima todo el movimiento por el deseo que inspira. Es el bien y el supremo deseable. El deseo es deseo de la presencia. El éros también es pensado a partir de la presencia. Como el movimiento. ${ }^{33}$

Sentido y presencia están onto-teleológicamente entrelazados, sea como «significación del discurso», sea en términos de «esencia», sea a nivel de «arkhe» o «telos»: el heideggeriano «aparecer con sentido» muestra esta íntima complicidad, a la cual pertenece la misma historia de la metafísica, entre el hacerse-presente y su inteligibilidad, entre la presencia y (la necesidad de) su sentido:

Ningún sentido [...] ha podido ser pensado en la historia de la metafísica de otro modo más que a partir de la presencia y como presencia. El concepto metafísico es gobernado por todo el sistema de determinaciones que aquí señalamos $\mathrm{y}$, cada vez que es planteada una cuestión de sentido, no puede serlo sino en el cierre metafísico. Sería, pues, vano, digámoslo seca y rápidamente, querer arrancar en tanto que tal la cuestión del sentido (del tiempo o de lo que sea) a la metafísica [...] Igualmente sucedería con una cuestión del ser que fuera determinada, como lo es en la apertura de Sein und Zeit, como cuestión del sentido del ser, cualquiera que sea la fuerza, la necesidad y el valor, tanto irruptora como de fundamento, de una cuestión semejante. En tanto que cuestión del sentido está ya ligada, en su punto de partida, y Heidegger lo reconocerá sin duda, al discurso (léxico y gramatical) de la metafísica cuya destrucción comienza. ${ }^{34}$

Derrida reconoce los esfuerzos heideggerianos por romper con una tradición filosófica que ha reducido el ser al ente, dejando así de pensar la verdad en términos de acontecimiento, de tensión y conflicto entre lo que se esconde y lo que empuja para salir a la luz. No menos importante ha sido el rechazo del dualismo platónico entre lo ideal y lo material, así como la necesidad de no concebir la experiencia de imagen en términos de semejanza o copia sensible de una realidad inteligible, modelo con el que conformarse. Asimismo, la atención sobre la capacidad, por parte de la imaginación, de realizar aquel doble movimiento de hacer-presente lo que no-está y actuar como puente entre lo sensible y lo a priori, ha permitido a Heidegger romper con las cadenas conceptuales en las que parecía quedar atado al pensamiento metafísico.

Con todo, nos recuerda Derrida, la escritura heideggeriana sigue siendo metafísica, sigue pendiente de la lógica binaria de las oposiciones semánticas, de lo «inauténtico» frente a lo «auténtico», de la «temporalidad originaria» frente

33 Márgenes de la filosofía, loc. cit., p. 86.

34 Ibid., pp. 85-86. 
a la «temporalidad destituida». Bajo esta perspectiva, resulta emblemática la expresión «exhibición originaria», cuya oposición frente a lo «derivado» no puede sino jugarse dentro del terreno de la metafísica:

Ahora bien, la oposición de lo originario y de lo derivado ¿no sigue siendo metafísica? La demanda de la arkhe en general, cualesquiera que sean las precauciones de las que se rodee a este concepto, ¿no es la operación "esencial” de la metafísica? ${ }^{35}$

Los planteamientos teóricos de Heidegger, por lo tanto, no parecen sugerir un camino alternativo a las estructuras dicotómicas del pensamiento occidental, todavía enredado en categorías semánticas que impiden el desarrollo de un pensamiento icónico capaz de emanciparse frente a la autoridad de la tradición logocéntrica.

\section{Lo Diferencial-DE-LA-IMAgen: Michals y DerRida.}

Una posible manera de salir del laberinto de la metafísica consistiría, quizás, en trasladar el enfoque del discurso filosófico, de la «comprensibilidad» al «pensamiento de la diferencia», del «aparecer con sentido» ${ }^{36}$ al acontecer diferencial. La oposición misma entre sentido y no-sentido podría sobrepasarse centrando la atención sobre el movimiento del diferir intrínseco a todo acontecimiento y a toda producción de significado, incluso a la distinción entre «el ser y lo que es», haciéndonos más bien pensar «en una escritura sin presencia y sin ausencia, sin historia, sin causa, sin arkhe, sin telos, una escritura que descompone absolutamente toda dialéctica, toda teología, toda teleología, toda ontología». ${ }^{37}$

Probablemente, en camino para acercarnos más a este movimiento del diferir, expresado por el concepto de acontecer diferencial, puede vislumbrarse también en el arte de Duane Michals, especialmente en la interpretación que Gloria Inés Ocampo Ramírez hace de la obra Una historia acerca de una historia - una imagen en blanco y negro, realizada en 1989, y parte de la serie "Textos". El tema de la imagen es representado por dos hombres: un primero que, en actitud pensativa nos mira y, a la vez, mira a sí mismo; frente a éste, un segundo personaje que, a pesar de la misma actitud y del mismo vestido del primero, se queda de espaldas respecto al espectador. Pues bien, a partir de un espejo colgado a la pared, detrás del primer personaje, surge un juego óptico de rebotes que, añadiéndose mutuamente a la escena central, hace que a una única y simple mirada se sobreponga la visual de «una prolongación cuasi

35 Ibid., p. 98.

36 «La reciente filosofía de la imagen. Análisis crítico del debate actual y consideración de posibles aportes», loc. cit., p. 283.

37 Ibid., p. 102. 
infinita de este hombre, cuasi infinita porque su único límite es el borde del encuadre fotográfico». ${ }^{38}$ Aquí, la dynamis de la imagen hace que el afuera y el adentro, el interior y el exterior se confundan en un juego de reflejos, en virtud del cual la subjetividad del protagonista se fragmenta en una multiplicidad de perspectivas que envuelve y condiciona tanto al hombre de la escena como al espectador. Quedamos atropellados por un «inagotable entramado de pluralidades similares, una inmensa disparidad en medio de lo uno, de lo heterogéneo» $\mathrm{y}$ «donde el centro y la periferia, al mismo tiempo son el núcleo, la médula». ${ }^{39}$

Ahora bien, el carácter arbitrario y - en cierta medida - ideológico de la dicotomía adentro/afuera queda subrayado por la teoría derridiana de la escritura, puesto que ésta «significa inscripción y ante todo institución durable de un signo», posibilidad de inscribir un espacio significante, anterior a toda oposición binaria ${ }^{40}$. Bajo esta perspectiva, la imagen misma es inscripción, capacidad de dejar huellas, «donde se marca la relación con lo otro», sin - antes de - que este otro venga entendido en oposición frente a lo mismo. ${ }^{41} \mathrm{La}$ dynamis de la imagen, en su hacer confundir el afuera con el adentro, coincide con la archi-escritura como movimiento del diferir a partir del cual toda distinción resulta posible. Precisamente en esta tensión entre los términos en conflicto se produce la posibilidad misma de todo efecto significante.

Sin embargo, para que ello se realice, es necesario que esta fuerza tensional se quede al margen de la elección entre un término y otro. Solamente en el juego inestable e indefinido de las oposiciones puede generarse sentido; el conflicto tiene que permanecer no-solucionado, no-apaciguado, ya que todo apaciguamiento implica violencia, implica sistemas jerárquicos de clasificación conceptual que obligan a una configuración espacio-temporal pre-constituida, intenta establecer de antemano la dirección de nuestros discursos, de nuestras miradas, de nuestros pensamientos.

Pues bien, frente a este totalitarismo del logos, a esta violencia metafísica que - tanto en Levinas como en Derrida - «en su sentido profundo estaría ligada a la opresión y el totalitarismo de lo mismo», en una «vieja complicidad entre la objetividad teórica y la posesión técnico-política», ${ }^{42}$ hay que pensar y actuar nietzscheanamente, afirmando la imposibilidad de toda clase de sistematización conceptual, «sin nostalgia», más bien «con una risa y un paso de danza». ${ }^{43}$

38 Gloria Inés Ocampo Ramírez, «Una historia acerca de una historia», Festival internacional de la imagen, XIII foro académico del diseño, 9-13 mayo/2016, Manizales-Colombia.

39 Ibid.

40 De la gramatología, op. cit., p. 58.

41 Ibid., p. 61.

42 Id. [1967c], La escritura y la diferencia. Barcelona: Anthropos, 2012, p. 124.

43 Id., Márgenes de la filosofía, op. cit., p. 62. 


\section{REFLEXIONES CONCLUSIVAS.}

Los análisis de Rubio nos han permitido vislumbrar en los enfoques fenomenológico y hermenéutico importantes contribuciones hacia una aclaración de la experiencia icónica.

Sin embargo, y a pesar de sus específicos elementos de fortaleza teórica, ambas perspectivas se han visto cuestionadas a la hora de enfrentarse a la teoría derridiana de la escritura. Ésta, en su declinación hacia el problema-imagen, ha operado un doble movimiento deconstructor.

Por un lado, ha opuesto a la seguridad trascendental de la presencia-a-sí ante la intuición, la necesidad de lo ausente, del todavía-no-presente en tanto su misma condición de aparecer.

Por otro lado, el heideggeriano horizonte de comprensibilidad en el que la imagen traería a la luz lo anteriormente-oculto, ha sido deslegitimado en sus presupuestos metafísicos, aún enredados en la dicotomía entre auténtico e inauténtico, entre originario y derivado.

La referencia al arte de Duane Michals nos ha otorgado las necesarias herramientas teóricas para vislumbrar en la filosofía derridiana el vehículo privilegiado de un pensamiento icónico capaz de romper con las directrices de la 'metafísica de la presencia'. Entendido como movimiento-del-diferir, independiente respecto a toda lógica binaria y a toda jerarquía dicotómica, el 'pensamiento de la diferencia' y el 'acontecer diferencial' nos han invitado a abordar el debate actual en filosofía de la imagen bajo una perspectiva capaz de unir sinérgicamente la alteridad, lo ausente, lo no-idéntico-consigo-mismo, con la producción de todo efecto semántico posible. Precisamente en esta línea de estudio, la imagen (como) différance podrá abrirnos innovadores y cada vez más fecundos caminos de investigación.

\section{REFERENCIAS BIBLIOGRÁFICAS.}

BÁEZ RUBÍ, L. 2010. «Reflexiones en torno a las teorías de la imagen en Alemania: la contribución de Klaus Sachs-Hombach», Anales del Instituto de Investigaciones Estéticas. UNAM, 97.

DERRIDA, J. 1993 [1967a]. La voz y el fenómeno. Introducción al problema del signo en la fenomenología de Husserl, tr. y prólogo Francisco Peñalver. Pre-Textos, Valencia: Pre-Textos.

___ 2003 [1967b]. De la gramatología, tr. Oscar del Barco y Conrado Ceretti. Colombia: Siglo XXI.

—__, 2012 [1967c]. La escritura y la diferencia, tr. Patricio Peñalver. Barcelona: Anthropos.

___ 2008 [1972a]. Márgenes de la filosofia, tr. Carmen Gonzáles Marín. Madrid: Cátedra. 
___ 2014 [1972b]. Posiciones. Entrevista con Jacques Derrida, tr. y prólogo Manuel Arranz. Valencia: Pre-Textos.

___ 2017 [1990]. Memorias de ciego, tr. Carlos Fisgativa. Bogotá: Uniandes, 2017. GARCÍA VARAS, A. (Editor) 2011. Filosofía de la imagen. Salamanca: Universidad de Salamanca.

HEIDEGGER, M. 2012 [1977]. El origen de la obra de arte. Caminos de bosque, tr. Helena Cortés y Artuto Leyte. Madrid: Alianza.

___ 2009 [1979]. Ser y Tiempo, tr. Jorge Eduardo Rivera. Madrid: Trotta.

OCAMPO RAMÍREZ, G. I. 2016. «Una historia acerca de una historia», Festival internacional de la imagen, XIII foro académico del diseño, 9-13 mayo, ManizalesColombia.

RUBIO, R. 2010. «La concepción ontológica de Heidegger sobre la producción», Gregorianum, 91, 2, pp. 343-369.

___ _ 2015. «El lugar de la fenomenología en el debate de la reciente filosofía de la imagen», Veritas, 33, pp. 89-101.

____ 2017. «La reciente filosofía de la imagen. Análisis crítico del debate actual y consideración de posibles aportes», Ideas y Valores, 66.163, pp. 273-298.

WIESING, L. 2000. Phänomene im Bild. Fink, München.

, Artifizielle Präsenz. Studien zur Philosophie des Bildes. Suhrkamp, Frankfurt a. M. 2005.

Jacopo Vignola es doctorando de la Universidad Alberto Hurtado (Santiago de Chile), actualmente se encuentra en la Università di Salerno (Italia)

Lineas de investigación:

Estética; Deconstrucción; Teoría Crítica

Publicaciones recientes:

Sulla propia pelle. La questione trascendentale tra Kant e Deleuze (con P. Vignola). Roma: ARACNE, 2012. ISBN: 978-88-548-4808-5

Trascendenze immanenti. Da Kant a Marcuse. Salerno: ORTHOTES, 2018. ISBN: 978$88-9314-126-0$

Email: vignolajacopo@gmail.com 
